\title{
退化羊草草原在浅耕翻处理后植物 群落演替动态研究
}

\section{宝音陶格涛 ${ }^{12}$ 刘美玲 2 李晓兰 2}

( 1 中国科学院植物研究所植被数量生态学重点实验室, 北京 100093) ( 2 内蒙古大学生态与环境科学系 , 呼和浩特 010021)

摘 要 在中国科学院生态中心草原站利用统计方法研究了退化羊草 (Leymus chinensis) 草原浅耕翻后群落演替规 律。通过对 18 年数据的分析结果表明:由于过度放牧而退化的羊草草原在浅耕翻处理后群落密度恢复较快,尤 其是羊草与处理前相比已有很大变化, 从而使群落得到了恢复。群落植物种的多样性、均匀性指数在这 18 年中均 呈抛物线状分布。羊草、冰草 (Agropyron michnoi)、变蒿(Artemisia commutata)、黄蒿 (A. scoparia)、星毛委陵菜 (Potentilla acaulia ) 的生物量与多样性指数和均匀性指数有显著的相关性。羊草的相对密度在第五年达到最高水平, 然后逐 渐下降, 自第十五年后羊草的相对密度基本趋于平衡, 其重要值呈单峰型曲线。退化羊草草原 18 年恢复过程可划 分为 4 个阶段 :第一阶段 (1 3 年) 为根茎禾草 + 一、二年生杂类草群落, 第二阶段 ( $4 \sim 9$ 年) 为纯羊草群落, 第三阶 段 (10 13 年) 为羊草 + 冰草 + 多年生杂类草过度型群落, 第四阶段 (14 18 年) 为羊草 + 冰草 + 丛生禾草、多年生 杂类草群落。经过处理后 18 年的恢复演替, 退化群落仍未恢复到原生群落, 演替将继续进行。

关键词 退化羊草草原 浅耕翻 植物群落 演替动态

\section{THE STUDY ON DYNAMICS SUCCESSION OF COMMUNITY IN DEGENERATED STEPPE OF LEYMUS CHINENSIS AFTER SHALLOW PLOUGHING}

\author{
BAOYIN Taogetao ${ }^{12}$ LIU Mei-Ling ${ }^{2}$ and LI Xiao-Lan ${ }^{2}$ \\ (1 Laboratory of Quantitative Vegetation Ecology, Institute of Botany, the Chinese Academy of Sciences , Beijing 100093 , China) \\ (2 Department of Ecological and Environmental Science, Inner Mongolia University , Huhhot 010021 , China)
}

\begin{abstract}
The study was conducted at Inner Mongolia Grassland Ecosystem Research Station of the Chinese Academy of Sciences in Xilingol Region, $43^{\circ} 20^{\prime}-44^{\circ} 00^{\prime} \mathrm{N}, 116^{\circ} 06^{\prime}-117^{\circ} 05^{\prime} \mathrm{E}$. The goal of the study was to provide some scientific basis for the restoration of degenerated grassland. Many statistical methods were used to study the succession laws of degenerated steppe of Leymus chinensis after shallow ploughing. After the data over a period of 18 years were analyzed, the results showed that the method of shallow ploughing could improve community density in degenerated steppe of $L$. chinensis due to overgrazing. The index of diversity and homogeneity of plant species resulted in a open-up parabola for 18 years. The relative density reached maximum at the fifth year, then gradually decreased. It tended to horizon since the fifteenth year. The important value of L. chinensis appeared open-down parabola. The process of community succession can be divided into four stages. The first stage was a community of $L$. chinensis + annual and biennial synusia (1-3 years after shallow ploughing). The constructive synusia was rhizome grass. Dominant synusia were annual and biennial synusia. The second stage was a community of $L$. chinensis (4-9 years after shallow ploughing). The constructive synusia was rhizome grass. Dominant synusia were thick grass and rhizome Carex. The third stage was a transition community of L. chinensis + Agropyron michnoi + rosette grasses (10-13 years after shallow ploughing). The constructive synusia was rhizome grass. Dominant synusia were short thick grass and rosette grass. The fourth stage was a community of $L$. chinensis $+A$. michnoi, thick grasses , rosette grasses (14-18 years after shallow ploughing). The constructive synusia was rhizome grass. Dominant synusia were thick grass and rosette grass. Through 18 years of restoration, the degenerated steppe was different from the primary communities of $L$. chinensis. Therefore, it is predicted that succession will continue until a stable community establishes.
\end{abstract}

Key words Degenerated steppe, L. chinensis, Shallow ploughing, Succession dynamics 
特有, 羊草草原群系广泛分布在我国东北、内蒙古东 部, 是在森林草原带与典型草原带的偏湿润一侧分 布面积很广的草原群系之一, 是发展畜牧业的物质 基础(李博等,1988 聂素梅等,1991)。长期以来,由 于人们对草地的经营管理不善,尤其是由于过度的 放牧利用，导致草原不同程度的退化，使草场生产力 和质量均有不同程度的下降。为了探索和寻求改良 退化羊草草原、提高群落生产力和恢复草地群落的 有效途径和方法，人们做了很大的努力并设计和使 用了许多方法 , 例如 ,围栏封育、松土改良(包括轻耙 处理和浅耕翻处理)、补播羊草等。本文的研究对象 是采用浅耕翻处理后恢复演替了 18 年的放牧退化 草场样地。该退化草地处理前为羊草草原群落因过 度放牧而形成的退化变体。群落为含小叶锦鸡儿 ( Caragana microphylla) 灌丛的冷蒿 (Artemisia frigida) + 羊草 + 丛生小禾草群落。虽然以前曾经有许多关 于浅耕翻处理后羊草草原恢复情况的文章 (马志广, 1982 ;宝音陶格涛等, 1992; 1996; 1997; 1999; 陈敏 等, 1997, 保平 ,1998 聂素梅, 1986 聂素梅等,1991)， 但上述研究都是针对草地质量变化及生产力水平的 研究, 且观测年限较短。而本文是对 18 年监测结果 的研究，对种类组成、群落密度及多样性、均匀性、重 要值都进行了分析, 并用 18 年数据结合群落组成等 指标研究了群落结构的变化情况和植物群落动态演 替规律 划分了演替阶段。进行本项研究，除了可为 退化草地的恢复改良提供依据外,其长期演替的研 究还可为退化草地的恢复演替理论提供依据。放牧 退化草地恢复的基本理论依据是 Clements 的 顶极 和植物演替理论”。但有关恢复演替的过程和演替 轨迹则存在着单稳态模式 (Mono-stable state) (Dysterhuis ,1949) 和复合稳态模式及阀值 (Metastablestates) (Hobbs \& Norton , 1996) 两种观点。同时组成群落的 有关个体的行为特征, 群落的功能群组成更是演替 研究不可缺少的内容 (Tilman,1994;Grime,1997a ; $1997 b$;王炜等, 2000;白永飞等,2001)。本研究的长 期监测结果既可为恢复演替的模式提供实例, 又通 过个体行为的动态研究为种群演替轨迹提供科学依 据。

\section{1 实验样地概况与研究方法}

\section{1 样地概况}

研究在内蒙古锡林郭勒盟白音锡勒牧场中国科 学院内蒙古草原生态系统定位研究站退化样地内进 行 研究区域位于 $43^{\circ} 30^{\prime} \sim 48^{\circ} 30^{\prime} \mathrm{N}, 113^{\circ} 30^{\prime} \sim 117^{\circ}$
30' E。本地区属于温带半干旱大陆性气候(温带草 原气候)，年均温 $-0.4{ }^{\circ} \mathrm{C}$, 年降水量 $300 \sim 400 \mathrm{~mm}$, 雨量多集中在 $6 \sim 8$ 月, 占全年降水量的 $70 \%$, 且年 蒸发量大于降雨量。本样地的土壤类型为砂质栗钙 土(陈佐忠, 1988; 陈敏等,1989)。处理前植被为羊 草 + 大针茅草原, 由于过度放牧而导致退化变体, 即 含小叶锦鸡儿灌丛的冷蒿 + 丛生小禾草 + 羊草群 落。草群低矮, 垂直成层性不明显, 盖度为 $30 \%$, 1983 年产草量是 $546 \mathrm{~kg} \mathrm{hm}^{-2}$ 。

\section{2 研究方法}

\subsection{1 处理和取样方法}

实验处理开始于 1983 年 7 月, 用机引四铧犁对 退化草地进行耕翻 深度 $18 \sim 20 \mathrm{~cm}$ 。在 1984 2000 年间, 每年 7 月和 8 月两次在固定区域用样方法调 查种群高度、密度和地上生物量等群落特征指标, 每 次 10 个重复样方，对各种特征指标进行统计分析， 并在处理的同时以同类型退化草地同期围封后的自 然恢复群落作为对照。

\subsection{2 多样性指数}

Shannon-Wiener 指数

$H=-\Sigma P_{i} \ln P_{i}$

其中 , $P_{i}$ 是第 $i$ 种的概率 $(\operatorname{Cox}, 1979)$ 。

\subsection{3 均匀度指数}

$$
E=H / \ln S
$$

其中 $H$ 为 Shannon-Wiener 指数, $S$ 为样方中植物种 数。

\subsection{4 重要值}

$$
I=R n+R w+R f
$$

其中 $R w 、 R n 、 R f$ 分别为相对生物量、相对密度、相 对频度，一个群落中所有种的重要值之和为 300。

\section{2 研究结果与分析}

\section{1 土壤特征}

如表 1 所示，浅耕翻处理的土壤，经过 18 年的 恢复, 其土壤容重低于对照土壤, 且 $20 \sim 30 \mathrm{~cm}$ 深土 层差异较表层大。土壤孔隙度也表现出相似特点。 此结果表明浅耕翻处理对原土壤结构产生了严重的 破坏, 虽经过 18 年的恢复, 土壤结构仍和未经处理 自然恢复的对照土壤存在着差异, 且处理土壤优于 对照土壤。土壤水分含量也表现出处理群落优于对 照群落的特点。

\section{2 群落种类组成的变化}

如表 2、表 3 所示,退化羊草草原在浅耕翻处理 后的恢复过程中, 植物群落种类组成有明显地变化, 
表 1 浅耕翻处理与对照间土壤特征的比较

Table 1 The comparison on soil characteristic between shallow ploughing and control

\begin{tabular}{|c|c|c|c|c|c|c|}
\hline \multirow{2}{*}{$\begin{array}{c}\text { 土层深度 } \\
\text { Depth of soil }(\mathrm{cm})\end{array}$} & \multicolumn{2}{|c|}{$\begin{array}{c}\text { 土壤容重 }\left(\mathrm{g} \mathrm{cm}^{-3}\right) \\
\text { Bulk density of soil }\end{array}$} & \multicolumn{2}{|c|}{$\begin{array}{c}\text { 土壤孔隙度 } \\
\text { Porosity of soil( \%) }\end{array}$} & \multicolumn{2}{|c|}{$\begin{array}{c}\text { 土壤水分 } \\
\text { Moisture of soil (\%) }\end{array}$} \\
\hline & $\begin{array}{c}\text { 浅耕翻 } \\
\text { Shallow ploughing }\end{array}$ & $\begin{array}{c}\text { 对照 } \\
\text { Control }\end{array}$ & $\begin{array}{c}\text { 浅耕翻 } \\
\text { Shallow ploughing }\end{array}$ & $\begin{array}{c}\text { 对照 } \\
\text { Control }\end{array}$ & $\begin{array}{c}\text { 浅耕翻 } \\
\text { Shallow ploughing }\end{array}$ & $\begin{array}{c}\text { 对照 } \\
\text { Control }\end{array}$ \\
\hline $0 \sim 10$ & $1.07 \pm 0.11$ & $1.09 \pm 0.05$ & $60.11 \pm 4.15$ & $59.02 \pm 1.99$ & $16.00 \pm 2.12$ & $10.10 \pm 0.99$ \\
\hline $10 \sim 20$ & $1.28 \pm 0.12$ & $1.33 \pm 0.06$ & $51.69 \pm 4.50$ & $50.04 \pm 2.26$ & $9.91 \pm 1.76$ & $6.34 \pm 1.47$ \\
\hline $20 \sim 30$ & $1.40 \pm 0.09$ & $1.53 \pm 0.24$ & $46.83 \pm 3.40$ & $42.09 \pm 9.06$ & $5.62 \pm 0.89$ & $4.86 \pm 0.25$ \\
\hline
\end{tabular}

表 2 退化草原浅耕翻处理后群落种类组成

Table 2 The changes of species after shallow ploughing on degenerated steppe

\begin{tabular}{cccc}
\hline $\begin{array}{c}\text { 年份 } \\
\text { Year }\end{array}$ & $\begin{array}{c}\text { 科数 } \\
\text { Family } \\
\text { number }\end{array}$ & $\begin{array}{c}\text { 属数 } \\
\text { Genus } \\
\text { number }\end{array}$ & $\begin{array}{c}\text { 种数 } \\
\text { Species } \\
\text { number }\end{array}$ \\
\hline 1983 & 10 & 18 & 22 \\
1984 & 11 & 19 & 23 \\
1993 & 12 & 19 & 27 \\
2000 & 15 & 26 & 32 \\
\hline 2000 (对照 Control) & 11 & 20 & 26 \\
\hline
\end{tabular}

由 1983 年末处理前的 10 科 18 属 22 种 到处理后第 二年 (1984 年)的 11 科 19 属 23 种, 1993 年 12 科 19 属 27 种，到 2000 年 15 科 26 属 32 种,分别比 1983 年增加了 $50 \% 、 44 \%$ 和 $45 \%$ 。处理后总的趋势是种 类组成有所增加。处理前, 群落中禾本科含 5 属 5 种居首位, 其次为豆科(含 3 属 3 种)、菊科(含 2 属 3 种)、蓄薇科(含 1 属 3 种)、藜科(含 2 属 2 种)。在 处理后前 3 年, 由于植被和土壤结构遭受耕翻扰动， 地表原有的植被受到破坏, 使得一、二年生植物 (以 藜科、菊科植物为主) 大量侵入, 在群落中占有很大 比例，其中处理后第二年 (1984 年) 愁科含 4 属 5 种 居首位 其次为菊科(含 2 属 4 种)、禾本科(含 3 属 3 种)、豆科(含 2 属 2 种)。其中藜科植物中以灰绿僽 (Chenopodium glaucum) 和猪毛菜 (Salsola collina) 所 占比例最大, 大约占群落总生物量的 $30 \%$ 。菊科植 物中主要以蒿属 (Artemisia) 植物为主。随着恢复过 程的延续，一二年生植物逐渐被多年生植物代替。 在处理后 $16 、 17$ 年时僽科植物和菊科植物又有增加 趋势, 因为此时羊草种群规模已经更趋于原生群落, 即处于向原生草原群落的演替阶段, 在单位面积内 种的丰富度也有所增加。到 2000 年禾本科含 5 属 5 种居首位, 其次是菊科(含 2 属 5 种)、僽科(含 3 属 4 种)、豆科(含 3 属 3 种)、百合科 (含 1 属 3 种)、菩薇 科(含 1 属 3 种)。与此同时, 各个种在群落中的比 例也发生了变化, 即发生了种的替代, 退化群落中原
来占优势的种群作用下降, 而与原生群落组成相同 的种类增加。群落组成逐渐向与原生群落相类似的 群落类型恢复。从植物功能群组成来看根茎禾草表 现出持续增加, 后期稍有回落的趋势, 且一直处于建 群的主导地位; 丛生禾草后期增加。一、二年生植物 在处理后的 $2 \sim 3$ 年所占比例较高, 然后迅速降低。

\section{3 群落密度年度变化}

如图 1 所示,在退化草原浅耕翻处理后 18 年的 恢复过程中群落密度变化呈先上升再回落又上升接 着又回落的多峰型变化趋势, 表现出密度变化的波 动性特点。其中僽科植物特别是猪毛菜、灰绿僽、刺 穗僽 (Chenopodium aristatum) 在处理后的 3 年内大量 增加, 形成了群落密度的第一个高峰期, 然后随着演

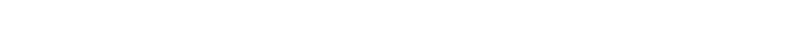
为根茎禾草在浅耕翻时根茎被切断, 从而刺激了地 下更新芽的生长, 随着根茎禾草羊草地上枝条逐渐 增加, 使得群落总密度随后几年呈现逐年增加的趋 势。从调查数据可知, 羊草由处理前 (1983 年) 的 44 株 $\mathrm{m}^{-2}$ 增加到处理后第二年 (1984 年) 的 212 株 $\mathrm{m}^{-2}$,1986 年又增长到 380 株 $\mathrm{m}^{-2}$ 此时已经较 1983 年增长了 $760 \%$,较 1984 年增长了 $70 \%$ 。而到了 1988 年竟达 520 株 $\mathrm{m}^{-2}$, 占群落密度的 $90 \%$ 以上， 这时的群落羊草已占绝对优势, 近乎为纯羊草群 落”, 远远高于原生羊草草原群落和同期自然恢复的 退化群落中羊草的水平, 形成了密度的第二个高峰。 在演替后期, 由于羊草枝条数的下降, 群落总密度相 应也有所降低, 但此时其它多年生植物的密度有所 增加, 因此出现总密度的又一个高峰。由此可见, 浅 耕翻处理后群落得到了很好地恢复, 密度有了明显 的变化, 特别是羊草在不同的恢复阶段其密度所占 的比例是不相同的 致使群落组成、结构和外貌最终 发生了明显的变化。

\section{4 群落多样性的变化}

生态系统中生物多样性的维持是全球生物多样 性与生态系统管理的中心目标之一（West，1993）, 同 
表 3 退化草原浅耕翻处理后群落种类组成及各种群相对生物量 $(\%)$

Table 3 The changes of species and biomass after shallow ploughing on degenerated steppe

\begin{tabular}{|c|c|c|c|c|c|c|}
\hline 植物名称 Species & 1983 & 1984 & 1988 & 1993 & 2000 & 2000 (对照 Control) \\
\hline 羊草 Leymus chinensis & $65.0 \pm 29.3$ & $47.5 \pm 12.0$ & $95.5 \pm 3.4$ & $64.0 \pm 11.0$ & $47.0 \pm 9.1$ & $44.2 \pm 6.0$ \\
\hline 冰草 Agropyron michnoi & $3.6 \pm 2.1$ & $5.5 \pm 3.4$ & $1.2 \pm 0.3$ & $3.0 \pm 1.6$ & $11.6 \pm 2.8$ & $27.7 \pm 5.3$ \\
\hline 徙隐子草 Cleistogenes squarrosa & $10.9 \pm 1.2$ & 0 & $0.1 \pm 0.3$ & $0.9 \pm 0.4$ & $1.3 \pm 1.1$ & $9.0 \pm 5.5$ \\
\hline 口草 Koeleria cristata & $5.1 \pm 2.3$ & 0 & 0 & 0 & $1.1 \pm 0.2$ & $4.1 \pm 1.4$ \\
\hline 针茅 Stipa grandis & $2.3 \pm 1.7$ & 0 & 0 & 0 & $4.4 \pm 2.5$ & $7.0 \pm 1.5$ \\
\hline 苔草 Carex korshinskyi & $0.1 \pm 0.1$ & $2.2 \pm 1.7$ & $0.9 \pm 0.8$ & $3.5 \pm 1.6$ & $3.9 \pm 1.9$ & $10.1 \pm 6.8$ \\
\hline 双齿偬 Allium anisopodium & $12.0 \pm 2.3$ & $0.2 \pm 0.1$ & $0.2 \pm 0.1$ & $0.6 \pm 0.5$ & $0.5 \pm 0.4$ & $4.1 \pm 1.4$ \\
\hline 细叶葱 A. tenuissimum & $0.4 \pm 0.3$ & 0 & 0 & $0.1 \pm 0.1$ & $0.3 \pm 0.2$ & $0.9 \pm 0.5$ \\
\hline 山葱 $A$. senescens & 0 & 0 & 0 & $0.2 \pm 0.5$ & 0 & 0 \\
\hline 野非 A. ramosum & 0 & $1.4 \pm 0.7$ & $0.6 \pm 0.1$ & 0 & $0.1 \pm 0.2$ & $1.4 \pm 0.6$ \\
\hline 茑尾 Iris tenuifolia & 0 & 0 & $0.1 \pm 0.1$ & 0 & $0.1 \pm 0.1$ & 0 \\
\hline 变蒿 Artemisia commutata & $7.6 \pm 3.9$ & $0.2 \pm 0.4$ & 0 & $5.3 \pm 3.4$ & $2.2 \pm 1.4$ & $3.4 \pm 2.3$ \\
\hline 黄蒿 $A$. scoparia & 0 & 0 & $0.4 \pm 0.2$ & $8.4 \pm 8.3$ & $1.7 \pm 1.3$ & $0.4 \pm 0.3$ \\
\hline 冷蒿 $A$. frigida & $35.1 \pm 7.4$ & $0.2 \pm 0.2$ & $0.1 \pm 0.1$ & $1.7 \pm 0.8$ & $2.6 \pm 1.3$ & $40.1 \pm 16.6$ \\
\hline 阿尔泰狗娃花 Heteropappua altaicus & $0.3 \pm 0.2$ & 0 & $0.1 \pm 0.3$ & $3.9 \pm 3.6$ & $0.2 \pm 0.2$ & $2.2 \pm 1.3$ \\
\hline 大籽蒿 Artemisia sieversiana & 0 & 0 & 0 & 0 & $0.1 \pm 0.1$ & 0 \\
\hline 麻花头 Serratula centauroides & 0 & 0 & 0 & $0.1 \pm 0.4$ & 0 & 0 \\
\hline 锦鸡儿 Caragana microphylla & $4.6 \pm 3.1$ & 0 & $0.1 \pm 0.1$ & 0 & $2.5 \pm 1.8$ & $3.4 \pm 2.9$ \\
\hline 扁宿豆 Melissitus ruthenica & $3.6 \pm 3.1$ & $0.3 \pm 0.5$ & $0.1 \pm 0.1$ & $0.3 \pm 0.8$ & $0.3 \pm 0.8$ & $0.2 \pm 0.1$ \\
\hline 乳白花黄芪 Astragalus galactites & $0.2 \pm 0.2$ & $0.8 \pm 1.1$ & 0 & $0.3 \pm 0.2$ & $0.1 \pm 0.1$ & 0 \\
\hline 星毛委陵菜 Potentilla acaulis & $2.2 \pm 1.3$ & $0.1 \pm 0.2$ & $0.1 \pm 0.5$ & $0.6 \pm 0.8$ & $2.9 \pm 2.2$ & $7.8 \pm 2.2$ \\
\hline 菊叶委陵菜 $P$. tanacetifolia & $4.5 \pm 5.2$ & 0 & $0.1 \pm 0.1$ & 0 & $2.0 \pm 2.1$ & $4.2 \pm 4.3$ \\
\hline 二裂委陵菜 $P$. bifurca & $0.1 \pm 0.3$ & $1.1 \pm 1.1$ & $0.7 \pm 0.5$ & $3.4 \pm 3.1$ & $4.3 \pm 3.3$ & $2.7 \pm 2.6$ \\
\hline 唐松草 Thalictrum petaloideum & $0.1 \pm 0.2$ & $1.4 \pm 1.8$ & $0.2 \pm 0.3$ & $0.1 \pm 0.1$ & $0.3 \pm 0.4$ & $0.1 \pm 0.1$ \\
\hline 女娄菜 Melandrium apricum & 0 & $0.1 \pm 0.4$ & 0 & 0 & $0.2 \pm 0.7$ & $0.1 \pm 0.1$ \\
\hline 轴藜 Axyris amaranthoides & 0 & $0.1 \pm 0.1$ & 0 & 0 & $0.1 \pm 0.3$ & $0.1 \pm 0.2$ \\
\hline 灰绿藜 Chenopodium glaucum & $0.1 \pm 0.2$ & $10.7 \pm 6.6$ & 0 & $0.9 \pm 0.9$ & $0.7 \pm 1.7$ & $0.2 \pm 1.2$ \\
\hline 木地肤 Kochia prostrata & $0.8 \pm 1.1$ & 0 & 0 & 0 & $0.9 \pm 1.1$ & $1.4 \pm 1.2$ \\
\hline 猪毛菜 Salsola collina & 0 & $23.4 \pm 6.0$ & 0 & 0 & $1.6 \pm 1.0$ & 0 \\
\hline 刺穗藜 Chenpodium aristatum & 0 & $0.3 \pm 0.2$ & 0 & 0 & 0 & 0 \\
\hline 花旗竿 Dontostemon eglandulosum & $0.1 \pm 0.2$ & $3.5 \pm 3.8$ & 0 & $0.7 \pm 1.6$ & $0.1 \pm 0.1$ & $0.1 \pm 0.2$ \\
\hline 防风 Saposhnikovia divaricata & 0 & $0.2 \pm 0.3$ & $0.4 \pm 0.9$ & $1.0 \pm 0.6$ & $5.2 \pm 4.4$ & $0.1 \pm 0.2$ \\
\hline 田旋花 Convolvulus arvensis & 0 & $0.5 \pm 1.3$ & $0.4 \pm 0.1$ & 0 & 0 & 0 \\
\hline 鹤虫 Lappula redonskii & $0.1 \pm 0.3$ & 0 & 0 & 0 & $0.5 \pm 0.2$ & 0 \\
\hline 芯芭 Cymbaria dahurica & 0 & 0 & 0 & 0 & 0 & $1.9 \pm 0.7$ \\
\hline 二色补血草 Limonium bicolor & 0 & 0 & 0 & $0.9 \pm 0.3$ & $0.3 \pm 0.9$ & 0 \\
\hline
\end{tabular}

时 理论、实验研究也显示出生物多样性对草地生态 系统的变化过程有着重要影响和作用 ( Tilman, 1994)。因此，对人为扰动下恢复过程多样性及变化 规律的研究具有重要的理论和实践意义(白永飞等， 2000)。从图 2 可以看出在处理后第一年 (1984 年) 和处理后第七年 (1990 年),第十六年 (1999 年) 出现 多样性指数的 3 个高峰期。第一个高峰的产生是由 于处理初期和一、二年生植物的侵入, 尤其是莍科植 物占有一定的优势, 群落的均匀程度和丰富度较高。 随后一、二年生植物逐渐被原生群落中建群种、优势 种代替,所以形成了多样性的又一个高峰期。随着 恢复过程的延续，建群种羊草和优势种的优势度逐 渐增强, 各种群在群落中所占比例差距增加 ,多样性
指数下降。多样性指数在处理后的总变化趋势为开 口向上的抛物线状, 其回归方程为 $D I=1.624-$ $0.16 t+0.12 t^{2} \quad\left(R^{2}=0.621 \quad r_{0.01,16}=0.590\right)$, 式 中 $D I$ 为多样性指数, $t$ 为处理后的年限。这一结果 说明 物种多样性可作为丰富度与均匀度的一个综 合反映，在退化草地改良恢复过程中均匀度起着更 主导的作用。同时, 相关分析表明, 群落主要植物种 群生物量与多样性指数存在着明显的相关关系(图 4)。从图 4 中可以看出, 羊草、冰草 (Agropyron mich$n o i) 、$ 变蓠 (Artemisia commutata)、星毛委陵菜 (Potentilla acaulis ) 的生物量与多样性指数呈线性关系。

\section{5 群落均匀性的变化}

如图3所示,其均匀度的变化与多样性的变化 

曰不本科 Gramineae
豆科 Leguminosae
四莎草科 Cyperaceae
圈菊科 Compositae
田蓄薇科 Rosaceae
四㔨科 Chenopodiaceae

因其它科 Other families

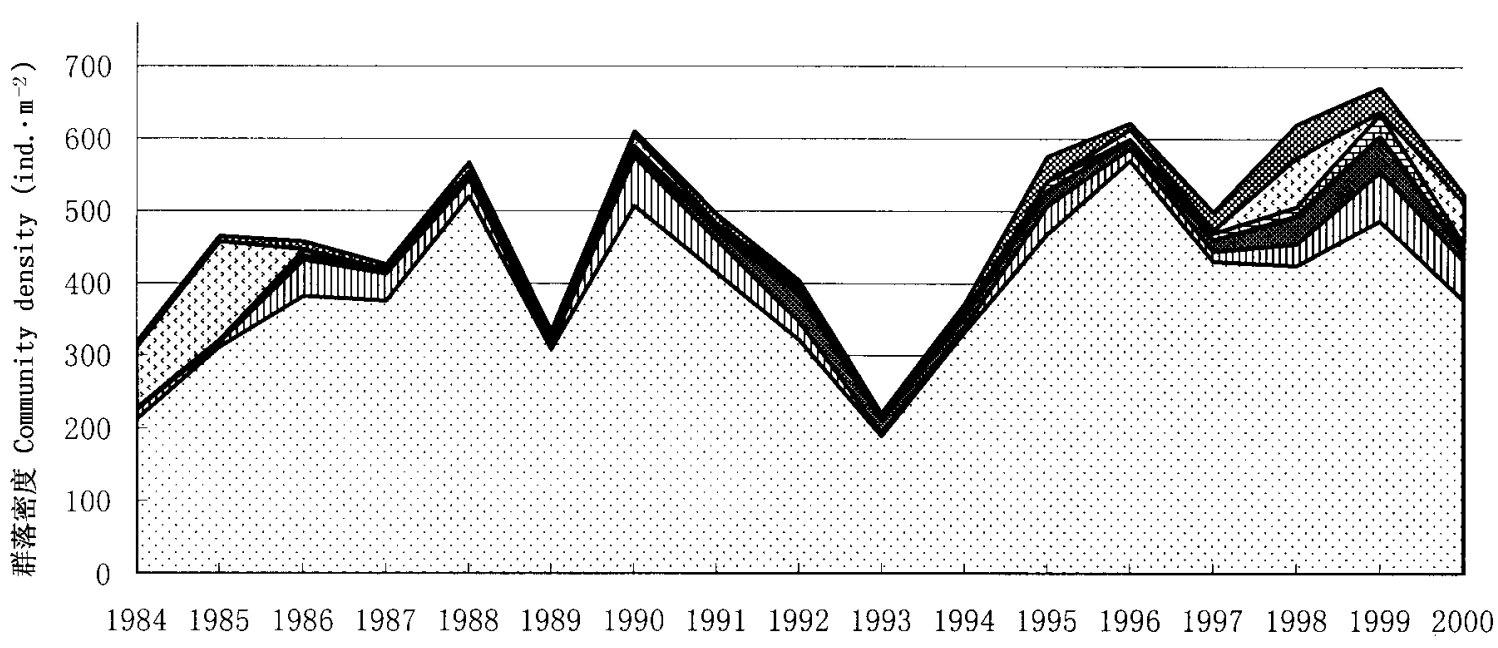

图 1 退化草原浅耕翻处理后演替过程中密度变化

Fig. 1 The change of density of degenerated steppe after shallow ploughing

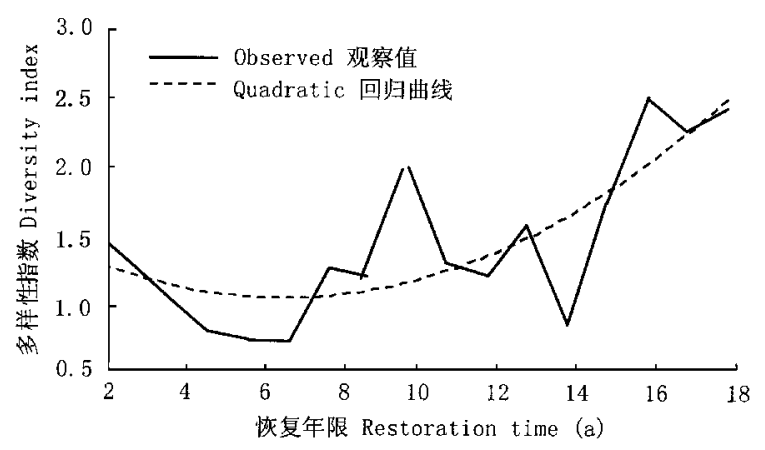

图 2 多样性指数在演替过程中的变化

Fig.2 The changes of diversity index during succession

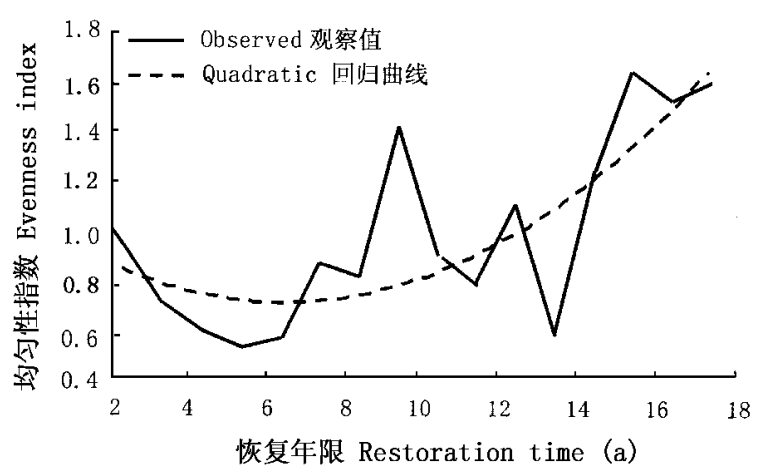

图 3 均匀性指数在演替过程中的变化

Fig. 3 The changes of evenness index during succession

相对应, 在浅耕翻处理后由于根茎禾草的根茎被切 断 刺激了这些种群增长, 因而使群落的均匀度下 降。在处理后第五年 (1988 年) 羊草种群增加到顶 峰, 个别种群的富集使均匀性和多样性指数降到最
低; 以后随着演替的进行, 刚处理时比较占优势的藜 科和菊科植物的作用逐渐被适应地带性气候条件的 禾草所代替, 羊草在草群中的比例下降, 群落的均匀 性和多样性指数有所回升, 以后羊草所占比例将基 本趋于稳定。此后的一段时间内僽科植物, 羊草、冰 草等禾草基本趋于一个稳定的状态, 所以均匀度又 呈现上升状态。这一现象表明原生草原群落中优势 种为羊草, 由于放牧等干扰羊草种群降低，从而造成 了处理前及处理初期的几年间群落的均匀度较高。 随着退化群落的恢复优势种群逐渐占据优势, 均匀 度也随之下降。羊草种群在整个演替过程中所占比 例由恢复前期的逐年上升到后期有所下降，所以整 体趋势是均匀度呈现一个开口向上的拋物线状, 其 回归方程为 $E I=1.16-0.11 t+0.01 t^{2} \quad\left(R^{2}=\right.$ $\left.0.574 r_{0.05,16}=0.482\right)$, 式中 $E I$ 为均匀性指数, $t$ 为处理后年限。

\section{6 主要种群重要值的变化}

重要值是用来表示某个种在群落中的地位和作 用的综合特征指标。植物种群作为群落的基本组成 成分, 它的动态变化规律在一定程度上反映了群落 的变化。特别是去除放牧干扰后退化群落的恢复过 程中种群的变化情况。为了描述种群在恢复演替过 程中的变化, 我们利用重要值这一指标选择了几种 主要植物种群，从其变化情况 (表 4) 可以看出:羊草 作为退化前的原生群落的建群种在退化草原群落中 并不占优势地位, 但经浅耕翻处理后, 使得原来因放 
羊草Leymus chinensis $r=-0.656$

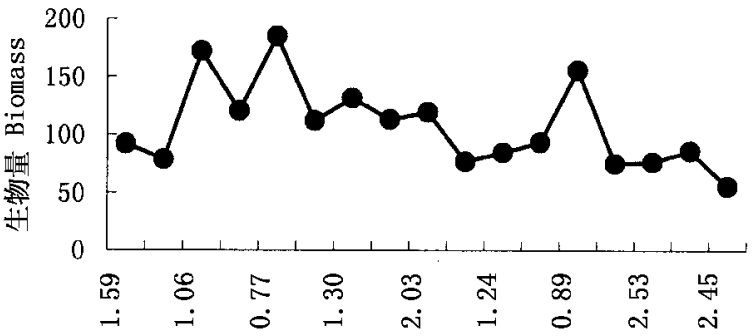

变蒿 Artemisia commutata $r=0.662$

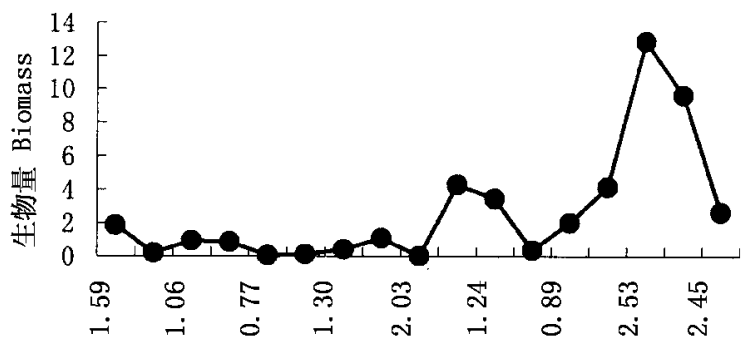

多样性指数 Diversity index
冰草 Agropyron michnoi $r=0.631$

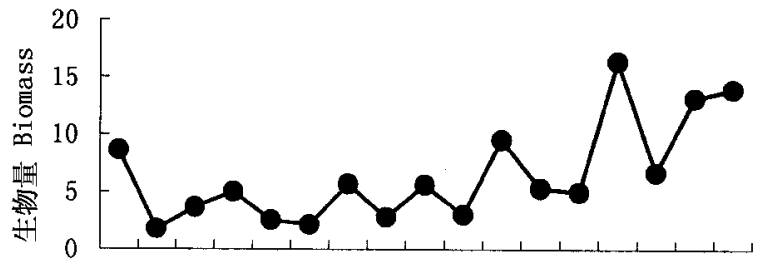

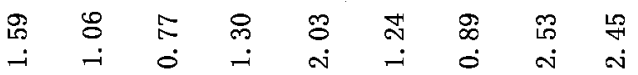

星毛委陵菜 Potentilla acaulia $r=0.64$

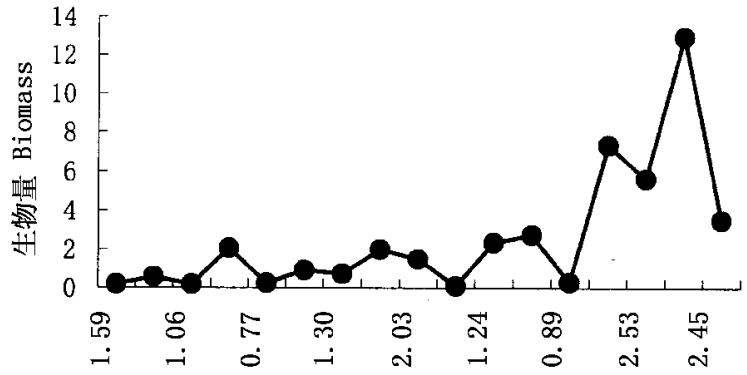

多样性指数 Diversity index

图 4 主要植物种群生物量与多样性指数的相关关系

Fig.4 The relationship of main plant population biomass and diversity $\left(r_{0.01,16}=0.590\right)$

表 4 退化羊草草原浅耕翻处理后各恢复阶段群落特征变化

Table 4 The characteristic changes of community in different restoration succession stages

\begin{tabular}{|c|c|c|c|c|}
\hline \multirow{2}{*}{$\begin{array}{l}\text { 群落特征 } \\
\text { Community } \\
\text { characteristics }\end{array}$} & \multicolumn{4}{|c|}{ 恢复演替阶段 Restoration succession stages } \\
\hline & $\begin{array}{l}\text { 第一阶段 } \\
\text { First stage }\end{array}$ & $\begin{array}{c}\text { 第二阶段 } \\
\text { Second stage }\end{array}$ & $\begin{array}{l}\text { 第三阶段 } \\
\text { Third stage }\end{array}$ & $\begin{array}{l}\text { 第四阶段 } \\
\text { Fourth stage }\end{array}$ \\
\hline $\begin{array}{l}\text { 恢复时间 Years after } \\
\text { shallow ploughing (a) }\end{array}$ & $\begin{array}{c}1-3 \text { years } \\
(1983-1985)\end{array}$ & $\begin{array}{c}4-9 \text { years } \\
(1986-1991)\end{array}$ & $\begin{array}{l}10-13 \text { years } \\
(1992-1995)\end{array}$ & $\begin{array}{l}14-18 \text { years } \\
(1996-2000)\end{array}$ \\
\hline $\begin{array}{l}\text { 群落类型 } \\
\text { Community } \\
\text { type }\end{array}$ & $\begin{array}{l}\text { 羊草 }+ \text { 一二年 } \\
\text { 生杂类草 } \\
\text { L. chinensis }+ \text { annual } \\
\text { or biennial forbs }\end{array}$ & $\begin{array}{l}\text { 羊草群落 } \\
\text { L. chinensis } \\
\text { community }\end{array}$ & $\begin{array}{c}\text { 羊草 }+ \text { 冰草 }+ \text { 多年生 } \\
\text { 杂类草过渡型群落 } \\
\text { L. chinensis }+ \text { A. michnoi }+ \\
\text { perennial forbs } \\
\text { transitional community }\end{array}$ & $\begin{array}{c}\text { 羊草 }+ \text { 冰草 }+ \text { 丛生禾草 } \\
\text { 多年生杂类草群落 } \\
\text { L. chinensis }+ \text { A. michnoi }+ \\
\text { bunch grass } \\
\text { community }\end{array}$ \\
\hline $\begin{array}{l}\text { 群落密度 } \\
\text { Density }\left(\text { No}_{0} ＼textrm{m}^{-2}\right)\end{array}$ & $300 \sim 450$ & $330 \sim 600$ & $220 \sim 620$ & $500 \sim 670$ \\
\hline $\begin{array}{l}\text { 地上生物量 } \\
\text { Aboveground biomass }\left(\mathrm{g} \mathrm{m}^{-2}\right)\end{array}$ & $100 \sim 219$ & $130 \sim 198$ & $115 \sim 185$ & $115 \sim 170$ \\
\hline 重要值 Importance value & & & & \\
\hline 羊草 Leymus chinensis & $118 \sim 155$ & $172 \sim 197$ & $160 \sim 180$ & $108 \sim 130$ \\
\hline 冰草 Agropyron michnoi & $7 \sim 12$ & $9 \sim 16$ & $11 \sim 60$ & $25 \sim 40$ \\
\hline 猪毛菜 Salsola collina & $4 \sim 25$ & $3 \sim 10$ & $0 \sim 8$ & $2 \sim 10$ \\
\hline 糙隐子草 Cleistogenes squarrosa & $0 \sim 1$ & $4 \sim 7$ & $5 \sim 9$ & $7 \sim 9$ \\
\hline 黄蒿 Artemisia scoparia & $2 \sim 3$ & $5 \sim 13$ & $6 \sim 30$ & $8 \sim 20$ \\
\hline 苔草 Carex korshiskyi & $12 \sim 13$ & $10 \sim 24$ & $7 \sim 16$ & $9.5 \sim 22$ \\
\hline
\end{tabular}

牧而硬结的土壤的通气透水性增加，同时又切断了 根茎禾草的部分根茎, 这对羊草种群的增长起了一 定的促进作用，地下芽萌发加快。所以羊草的重要 值在前几年持续快速增加, 特别是前五、六年羊草重
要值增长很快 然后其重要值趋于一个平缓的趋势。 与此同时，处理开始时占优势的一、二年生植物猪毛 菜、灰绿䔧呈现逐渐下降的趋势, 个别年份也会出现 但并不多。冰草作为恢复演替的优势种群, 其重要 
值呈现前期平缓后期加速的总体波动上升的趋势。 苔草 (Carex korshinskyi)、糙隐子草(Cleistogenes squarros $a$ )、星毛委陵菜、二裂委陵菜 ( P. bifurca $)$ 的重要 值基本趋于一个有起伏的相对缓慢上升状态。而退 化群落的建群种冷蒿在处理后迅速减少, 恢复过程 中已经处于伴生地位。

\section{7 植物群落的演替阶段}

结合群落结构及种群特征可以把 18 年的恢复 过程划分为 4 个演替阶段 (表 4)。第一阶段, 即处 理初期 ( 1983 1985), 羊草刚刚开始恢复, 群落中 一、二年生植物较多, 建群层片为根茎禾草层片, 优 势层片为一、二年生草本层片。群落类型为羊草 + 一、二年生杂类草。第二阶段, 即 1986 1991 年, 群 落为波动的逆行演替, 羊草种群增加, 在 1988 年达 到顶峰, 群落趋于纯羊草群落, 羊草的重要值由 172 上升到 197 , 建群层片为根茎禾草层片, 优势层片为 多年生杂类草、根茎苔草, 此阶段为羊草群落阶段。 第三阶段(1992 1995), 羊草种群相对下降, 群落演 替方向趋于正向演替, 此时群落的物种多样性、均匀 性有所增加, 羊草重要值略有下降, 建群层片根茎禾 草层片, 优势层片为小丛生禾草、多年生杂类草层 片, 形成了羊草 + 冰草 + 多年生杂类草的过渡型群 落类型。第四阶段，即演替后期（1996２000）, 此阶 段的建群层片根茎禾草层片，优势层片为丛生禾草、 多年生杂类草层片, 群落类型已完全成为羊草 + 冰 草 + 丛生禾草、多年生杂类草群落, 此时羊草的重要 值已基本上趋于原生群落。

\section{3 结论与讨论}

通过对浅耕翻处理后 18 年的退化草地恢复过 程的定位研究, 目前可得到如下阶段性结论:1) 羊草 草原由于过度放牧而退化，通过浅耕翻不仅提高了 羊草的密度, 而且使原退化群落得到不同程度恢复， 其群落种类组成和结构有所改变且生产力的恢复先 于群落结构的恢复。2) 由 18 年的观测可知, 退化 羊草草原群落的均匀性和多样性指数在浅耕翻处理 后先下降后上升然后又下降, 其回归方程为 $D I=$ $1.624-0.16 t+0.12 t^{2} \quad\left(R^{2}=0.621\right)$ 和 $E I=1.16-$ $0.11 t+0.01 t^{2} \quad\left(R^{2}=0.574\right)$ 植物群落多样性变化 主要取决于其物种的均匀性变化。3)处理后第五年 羊草的种群达最大值, 此时群落的组成结构也发生 了变化, 羊草的重要值与恢复时间的关系可用方程 $I V=78.04+34.2 t-3.2 t^{2}+0.08 t^{3}$ 表示。4) 退化 羊草草原 17 年恢复过程可划分为 4 个阶段，第一阶
段 ( $1 \sim 3$ 年) 为根茎禾草 + 一、二年生杂类草群落， 第二阶段 ( $4 \sim 9$ 年) 为纯羊草群落, 第三阶段 (10 13 年) 为羊草 + 冰草 + 多年生杂类草过渡型群落, 第四 阶段 (14 18 年) 为羊草 + 冰草 + 丛生禾草、多年生 杂类草群落。5)经过 18 年的恢复演替, 退化群落仍 未恢复到原生羊草草原群落, 演替将继续进行。

浅耕翻处理作为退化草地改良措施之一，长期 定位研究除得到上述结论外, 仍有下列问题应引起 注意。首先因其对地表原有植被破坏较厉害，所以 应用必须具备一定的条件, 即适用于草甸草原或水 分条件较好的典型草原地区, 以根茎型禾草为建群 种的退化草地类型, 如退化羊草草原。同时处理后 需要一定的封育保护, 以免在初期因干扰而影响其 效果。同时因生产力的恢复先于群落结构的恢复, 对草地改良措施的评价，不应只考虑其增产效果，应 与其对整个退化生态系统功能的恢复联系起来, 例 如群落结构、群落多样性等综合指标。因为上述指 标都对生态系统的过程产生影响并起到控制作用 (Grime ,1997a；1997b;Pimm,1993 ;白永飞等，2001）。 有关恢复演替的单稳态和多稳态问题, 就本实验目 前的结论来看, 仍基本符合单稳态理论, 即演替方向 是向着原生羊草草原方向恢复, 尽管在后期恢复速 率减缓，但并未表现出明显的稳态阶段。只是不同 的恢复阶段群落的组成、结构等发生了替代。种间 竞争是群落演替的内在原因 (Tilman,1994) ,在本试 验中就组成群落的物种而言, 羊草是本项措施处理 后恢复最快且在整个恢复演替过程中起双重作用的 种群，即前期的率先恢复以至种群数量超过了原生 羊草草原群落中羊草的水平并处于绝对优势地位， 而后期由于竞争的结果羊草种群下降, 直至种群更 接近于原生羊草草原群落。可否把羊草在恢复后期 的作用看作是对恢复演替的一种阻碍, 因为其它相 关研究结果表明, 在同类退化群落的自然恢复演替 过程中，羊草属于持续增长型种群(王炜等, 1999; 宝 音陶格涛等, 1996) , 而在人为干扰下羊草种群对群 落长期演替的作用尚待讨论。因为只有通过竞争降 低羊草的优势度才能使其它物种的优势度逐渐提 高, 使整个群落的组成和结构以及多样性和均匀性 更趋合理。

\section{参 考 文 献}

Bai, Y.F. (白永飞), Z.X.Xu(许志信) \& D.X. Li(李德新) 2000. Study on $\alpha$ diversity of four Stipa communities in Inner Mongolia plateau. Chinese Biodiversity (生物多样性), 8: 353 360. (in Chinese with English abstract)

Bai, Y. F. (白永飞), L. H. Li (李凌浩) \& J. H. Huang (黄建 
辉). 2001. The influence of plant diversity and functional composition on ecosystem stability of four Stipa community in Inner Mongolia plateau. Acta Botanica Sinica(植物学报), 43: 280 287. (in Chinese with English abstract)

Bao, P. (保平). 1998. Analysis about soil improvement for increasing output benefit in semi-dry grassland. Grassland of China (中国草地) , 4: 46 48. (in Chinese with English abstract)

Baoyin, T. G. T. (宝音陶格涛) \& M. Chen (陈敏). 1992. The study on dynamics restoration succession of community in degenerated steppe of Leymus chinensis after shallow ploughing. Acta Scientiarum Naturalium Universitatis Neimongol (内蒙古大学学 报), 23: $204 \sim 207$. (in Chinese with English abstract)

Baoyin, T. G.T. (宝音陶格涛)，M. Chen(陈敏)\& J.H. Liu(柳 景辉). 1996. The study on dynamics restoration succession of community in degenerated steppe of Leymus chinensis after enclosed. Acta Scientiarum Naturalium Universitatis Neimongol(内 蒙古大学学报), 27: 103 110. (in Chinese with English abstract)

Baoyin, T.G. T. (宝音陶格涛) \& M. Chen (陈敏). 1997. The studies of changes of plant diversity on degenerated steppe in enclosed process. Acta Scientiarum Naturalium Universitatis Neimongol(内蒙古大学学报), 28: $87 \sim 91$. (in Chinese with English abstract)

Baoyin,T.G. T. (宝音陶格涛) \& Gel Cheng (成格尔). 1999. The study on restoration succession of community on degenerated steppe of Leymus chinensis after shallow ploughing. Acta Scientiarum Naturalium Universitatis Neimongol (内蒙古大学学报), 30: $354 \sim 359$. (in Chinese with English abstract)

Chen, M. (陈敏) \& T. G. T. Baoyin (宝音陶格涛). 1989. Research on improving degenerative steppe and methods for increasing productivity of the steppe region. Acta Phytoecologica et Geobotanica Sinica (植物生态学与地植物学学报), 13: 379 386. (in Chinese with English abstract)

Chen, M. (陈敏) \& T. G. T. Baoyin (宝音陶格涛). 1997. Improvement of regressive grassland in semi-arid steppe region. Pratacultural Science(草业科学), 6: $27 \sim 29$. (in Chinese with English abstract)

Chen, Z.Z. (陈佐忠). 1988. The landform and climate in Xilin river basin. Research on Grassland Ecosystem(草原生态系统 研究), 3: 19 20. (in Chinese with English abstract)

Cox, G.W. (translated by Jiang, Y.X. (蒋有绪) ). 1979. Laboratory manual of general ecology. Beijing: Science Press. 121 123. (in Chinese)
Dysterhuis, E. J. 1949. Condition and management of rangeland based on quantitative ecology. Journal of Rangeland Management, 2: $104 \sim 115$.

Grime, J. P. 1997a. Biodiversity and ecosystem function: the debate deepens. Science, 277: $1260 \sim 1261$.

Grime, J. P. 1997b. Evidence for the existence of three primary strategies in plants and its relevance to ecological and evolutionary theory. The American Naturalist, 111: $1119 \sim 1144$.

Hobbs, R. J. \& D. A. Norton. 1996. Towards a conceptual framework for restoration ecology. Restoration Ecology, 4:93 110 .

Li, B. (李博), S.P. Yong(雍世鹏) \& Z. H. Li (李忠厚). 1988. The vegetation of Xilin river basin and its utilization. Research on Grassland Ecosystem(草原生态系统研究)，3: $84 \sim 183$. (in Chinese with English abstract)

Ma,Z. G. (马志广). 1982. The study about soil improvement grassland. Grassland of China (中国草地), 2: $31 \sim 34$. (in Chinese with English abstract)

Nie,S.M. (聂素梅)，Y.Q. Wang(王育青) \& Z.W. Yang(杨 志伟). 1991. A technology of shallow plowing to improve degenerated grassland. Grassland of China(中国草地), 4: 31 34. (in Chinese with English abstract)

Nie,S.M. (聂素梅) . 1986. Using the method of shallow ploughing to improve steppe in semi-dry grassland. Grassland of China(中 国草地), 4: 27 29. (in Chinese with English abstract)

Pimm, S. L. 1993. Biodiversity and balance of nature. In: Schulze, E. D. \& H. A. Mooney eds. Ecological studies. Vol. 99. Berlin: Springer-Verlag. $348 \sim 359$.

Tilman, D. 1994. Competition and biodiversity in spatially structured habitats. Ecology, 75: $2 \sim 16$.

Wang, W. (王炜), C.Z. Liang (梁存柱) \& Z. L. Liu(刘钟龄). 1999. Research on restoring succession of degenerated grassland in Inner Mongolia. IV . Analysis of plant population dynamics during restoring succession. Journal of Arid Land Resources and Environment (干旱区资源与环境)，13(4)：44～55. (in Chinese with English abstract)

Wang, W. (王炜) , C.Z. Liang (粱存柱) \& Z. L. Liu(刘钟龄) . 2000. Analysis of the plant individual behaviour during the degradation and restoring succession in steppe community. Acta Phytoecologica Sinica (植物生态学报), 24: $268 \sim 274$. (in Chinese with English abstract)

West, N. E. 1993. Biodiversity of rangelands. Journal of Rangeland Management, 46: $2 \sim 13$. 